\title{
Generalized Relative Type and Generalized Weak Type of Entire Functions
}

\author{
Sanjib Kumar Datta, ${ }^{1}$ Tanmay Biswas, ${ }^{2}$ and Debasmita Dutta ${ }^{3}$ \\ ${ }^{1}$ Department of Mathematics, University of Kalyani, Nadia District, West Bengal 741235, India \\ ${ }^{2}$ Rajbari, Rabindrapalli, R. N. Tagore Road, Nadia District, West Bengal 741101, India \\ ${ }^{3}$ Mohanpara Nibedita Balika Vidyalaya (High), English Bazar Block, Malda District, West Bengal 732208, India \\ Correspondence should be addressed to Sanjib Kumar Datta; sanjib_kr_datta@yahoo.co.in
}

Received 29 November 2015; Accepted 28 March 2016

Academic Editor: Janne Heittokangas

Copyright (C) 2016 Sanjib Kumar Datta et al. This is an open access article distributed under the Creative Commons Attribution License, which permits unrestricted use, distribution, and reproduction in any medium, provided the original work is properly cited.

We study some relative growth properties of entire functions with respect to another entire function on the basis of generalized relative type and generalized relative weak type.

\section{Introduction}

A single valued function of one complex variable which is analytic in the finite complex plane is called an integral (entire) function. For example $\exp z, \sin z$, and $\cos z$ are examples of entire functions. In the value distribution theory one studies how an entire function assumes some values and the influence of assuming certain values in some specific manner on a function. In 1926 Rolf Nevanlinna initiated the value distribution theory of entire functions. This value distribution theory is a prominent branch of complex analysis and is the prime concern of the paper. Perhaps the Fundamental Theorem of Classical Algebra which states that "if $f$ is a polynomial of degree $n$ with real or complex coefficients, then the equation $f(z)=0$ has at least one root" is the most well known value distribution theorem.

The value distribution theory deals with various aspects of the behavior of entire functions one of which is the study of comparative growth properties. For any entire function $f$, the maximum modulus of $f$ is the function $M_{f}(r)$ defined as

$$
M_{f}(r)=\max _{|z|=r}|f(z)| .
$$

Similarly function $M_{g}(r)$ is defined for another entire function $g$. The ratio $M_{f}(r) / M_{g}(r)$ as $r \rightarrow \infty$ evaluates the growth of $f$ with respect to $g$ in terms of their maximum moduli.
However, the order of an entire function $f$ which is generally used in computational purpose is defined in terms of the growth of $f$ with respect to $\exp z$ function as

$$
\begin{aligned}
\rho_{f} & =\limsup _{r \rightarrow \infty} \frac{\log \log M_{f}(r)}{\log \log M_{\exp z}(r)} \\
& =\limsup _{r \rightarrow \infty} \frac{\log \log M_{f}(r)}{\log r} .
\end{aligned}
$$

Bernal $[1,2]$ introduced the relative order between two entire functions to avoid comparing growth just with $\exp z$, extending the notion of relative order as cit.op. Lahiri and Banerjee [3] introduced the definition of generalized relative order. In the case of generalized relative order, it therefore seems reasonable to define suitably the generalized relative type (generalized relative weak type) of an entire function with respect to another entire function in order to compare the relative growth of two entire functions having the same nonzero finite generalized relative order (generalized relative lower order) with respect to another entire function. In this connection Datta et al. [4] introduced the definition of generalized relative type (generalized relative weak type) of an entire function with respect to another entire function.

For entire functions, the notions of the growth indicators such as order and type (weak type) are classical in complex 
analysis and, during the past decades, several researchers have already been exploring them in the area of comparative growth properties of composite entire functions in different directions using the classical growth indicators. But, at that time, the concepts of relative order (generalized relative orders), relative type (generalized relative type), and relative weak type (generalized relative weak type) of entire functions and their technical advantages of not comparing with the growth of $\exp z$ are not at all known to the researchers of this area. Therefore the studies of the growth of composite entire functions in the light of their relative order (generalized relative orders), relative type (generalized relative type), and relative weak type (generalized relative weak type) are the main concern of this paper. In fact some light has already been thrown on such type of works by Datta et al. in [4-8]. Actually, in this paper, we study some relative growth properties of entire functions with respect to another entire function on the basis of generalized relative type and generalized relative weak type.

\section{Notation and Preliminary Remarks}

Our notations are standard within the theory of Nevanlinna's value distribution of entire functions and therefore we do not explain those in detail as available in [9]. In the sequel the following two notations are used:

$$
\begin{aligned}
& \log ^{[k]} x=\log \left(\log ^{[k-1]} x\right) \quad \text { for } k=1,2,3, \ldots ; \\
& \log ^{[0]} x=x, \\
& \exp ^{[k]} x=\exp \left(\exp ^{[k-1]} x\right) \quad \text { for } k=1,2,3, \ldots ; \\
& \exp ^{[0]} x=x .
\end{aligned}
$$

Taking this into account, Juneja et al. [10] defined the $(p, q)$ th order and $(p, q)$ th lower order of an entire function $f$, respectively, as follows:

$$
\begin{aligned}
& \rho_{f}(p, q)=\limsup _{r \rightarrow \infty} \frac{\log ^{[p]} M_{f}(r)}{\log ^{[q]} r}, \\
& \lambda_{f}(p, q)=\liminf _{r \rightarrow \infty} \frac{\log ^{[p]} M_{f}(r)}{\log ^{[q]} r},
\end{aligned}
$$

where $p, q$ are any two positive integers with $p \geq q$.

These definitions extended the definitions of $\operatorname{order} \rho_{f}$ and lower order $\lambda_{f}$ of an entire function $f$ which are classical in complex analysis for integers $p=2$ and $q=1$ since these correspond to the particular case $\rho_{f}(2,1)=\rho_{f}$ and $\lambda_{f}(2,1)=$ $\lambda_{f}$. Further, for $p=l$ and $q=1$, the above definitions reduce to generalized order $\rho_{f}^{[l]}$ [11] (resp., generalized lower order $\left.\lambda_{f}^{[l]}\right)$.
In this connection let us recall that if $0<\rho_{f}(p, q)<\infty$, then the following properties hold:

$$
\begin{aligned}
\rho_{f}(p-n, q)=\infty & \text { for } n<p, \\
\rho_{f}(p, q-n)=0 & \text { for } n<q, \\
\rho_{f}(p+n, q+n)=1 & \text { for } n=1,2, \ldots
\end{aligned}
$$

Similarly, for $0<\lambda_{f}(p, q)<\infty$, one can easily verify that

$$
\begin{aligned}
& \lambda_{f}(p-n, q)=\infty \text { for } n<p, \\
& \lambda_{f}(p, q-n)=0 \text { for } n<q, \\
& \lambda_{f}(p+n, q+n)=1 \quad \text { for } n=1,2, \ldots
\end{aligned}
$$

Recalling that for any pair of integer numbers $m, n$ the Kronecker function is defined by $\delta_{m, n}=1$ for $m=n$ and $\delta_{m, n}=0$ for $m \neq n$, the aforementioned properties provide the following definition.

Definition 1 (see [10]). An entire function $f$ is said to have index-pair $(1,1)$ if $0<\rho_{f}(1,1)<\infty$. Otherwise, $f$ is said to have index-pair $(p, q) \neq(1,1), p \geq q \geq 1$, if $\delta_{p-q, 0}<$ $\rho_{f}(p, q)<\infty$ and $\rho_{f}(p-1, q-1) \notin \mathbb{R}^{+}$.

Definition 2 (see [10]). An entire function $f$ is said to have lower index-pair $(1,1)$ if $0<\lambda_{f}(1,1)<\infty$. Otherwise, $f$ is said to have lower index-pair $(p, q) \neq(1,1), p \geq q \geq 1$, if $\delta_{p-q, 0}<\lambda_{f}(p, q)<\infty$ and $\lambda_{f}(p-1, q-1) \notin \mathbb{R}^{+}$.

Remark 3. An entire function $f$ of index-pair $(p, q)$ is said to be of regular $(p, q)$-growth if its $(p, q)$ th order coincides with its $(p, q)$ th lower order, otherwise $f$ is said to be of irregular $(p, q)$-growth.

To compare the growth of entire functions having the same $(p, q)$ th order, Juneja et al. [12] also introduced the concepts of $(p, q)$ th type and $(p, q)$ th lower type in the following manner.

Definition 4 (see $[12])$. The $(p, q)$ th type and the $(p, q)$ th lower type of entire function $f$ having finite positive $(p, q)$ th order $\rho_{f}(p, q)\left(b<\rho_{f}(p, q)<\infty\right)$ are defined as

$$
\begin{aligned}
& \sigma_{f}(p, q)=\limsup _{r \rightarrow \infty} \frac{\log ^{[p-1]} M_{f}(r)}{\left(\log ^{[q-1]} r\right)^{\rho_{f}(p, q)}}, \\
& \bar{\sigma}_{f}(p, q)=\liminf _{r \rightarrow \infty} \frac{\log ^{[p-1]} M_{f}(r)}{\left(\log ^{[q-1]} r\right)^{\rho_{f}(p, q)}}, \\
& \quad 0 \leq \bar{\sigma}_{f} \leq \sigma_{f} \leq \infty,
\end{aligned}
$$

where $p, q$ are any two positive integers, $b=1$ if $p=q$, and $b=0$ for $p>q$.

Remark 5. For $p=l$ and $q=1$, the above definitions reduce to generalized type $\sigma_{f}^{[l]}$ and generalized lower type $\bar{\sigma}_{f}^{[l]}$ of an 
entire function $f$. Moreover, when $p=2$ and $q=1$, then $\sigma_{f}^{[2]}$ and $\bar{\sigma}_{f}^{[2]}$ are correspondingly denoted as $\sigma_{f}$ and $\bar{\sigma}_{f}$ which are, respectively, known as type and lower type of entire $f$.

Now we introduce the following definitions in order to determine the relative growth of two entire functions having the same nonzero finite $(p, q)$ th lower order in the following manner.

Definition 6. The $(p, q)$ th weak type $\tau_{f}(p, q)$ and the growth indicator $\bar{\tau}_{f}(p, q)$ of an entire function $f$ having finite positive $(p, q)$ th lower order $\lambda_{f}(p, q)\left(b<\lambda_{f}(p, q)<\infty\right)$ are defined by

$$
\begin{aligned}
\tau_{f}(p, q) & =\liminf _{r \rightarrow \infty} \frac{\log ^{[p-1]} M_{f}(r)}{\left(\log ^{[q-1]} r\right)^{\lambda_{f}(p, q)}}, \\
\bar{\tau}_{f}(p, q) & =\limsup _{r \rightarrow \infty} \frac{\log ^{[p-1]} M_{f}(r)}{\left(\log ^{[q-1]} r\right)^{\lambda_{f}(p, q)}}, \\
& 0<\lambda_{f}(p, q)<\infty,
\end{aligned}
$$

where $p, q$ are any two positive integers, $b=1$ if $p=q$, and $b=0$ for $p>q$.

Remark 7. If we consider $p=l$ and $q=1$ in the above definitions, then the growth indicators $\tau_{f}(l, 1)$ and $\bar{\tau}_{f}(l, 1)$ are correspondingly denoted as $\tau_{f}^{[l]}$ and $\bar{\tau}_{f}^{[l]}$. Further, for $p=2$ and $q=1$, the above definition reduces to the classical definition as established by Datta and Jha [13]. Also $\tau_{f}$ and $\bar{\tau}_{f}$ stand for $\tau_{f}^{[2]}$ and $\bar{\tau}_{f}^{[2]}$.

For any two entire functions $f$ and $g$, Bernal $[1,2]$ initiated the definition of relative order of $f$ with respect to $g$, indicated by $\rho_{g}(f)$, as follows:

$$
\begin{aligned}
\rho_{g} & (f) \\
& =\inf \left\{\mu>0: M_{f}(r)<M_{g}\left(r^{\mu}\right), \forall r>r_{0}(\mu)>0\right\} \\
& =\limsup _{r \rightarrow \infty} \frac{\log M_{g}^{-1} M_{f}(r)}{\log r},
\end{aligned}
$$

which keeps away from comparing growth just with $\exp z$ to find out order of entire functions as we see earlier and of course this definition corresponds with the classical one [14] for $g=\exp z$.

Remark 8. In line with the above definition, one may define the relative lower order of $f$ with respect to $g$, denoted by $\lambda_{g}(f)$, as

$$
\lambda_{g}(f)=\liminf _{r \rightarrow \infty} \frac{\log M_{g}^{-1} M_{f}(r)}{\log r} .
$$

Extending this notion, Lahiri and Banerjee [3] gave a more generalized concept of relative order in the following way.
Definition 9 (see [3]). If $l \geq 1$ is a positive integer, then the $l$ th generalized relative order of $f$ with respect to $g$, denoted by $\rho_{g}^{[l]}(f)$, is defined by

$$
\begin{gathered}
\rho_{g}^{[l]}(f)=\inf \left\{\mu>0: M_{f}(r)<M_{g}\left(\exp ^{[l-1]} r^{\mu}\right), \forall r\right. \\
\left.>r_{0}(\mu)>0\right\}=\limsup _{r \rightarrow \infty} \frac{\log ^{[l]} M_{g}^{-1} M_{f}(r)}{\log r} .
\end{gathered}
$$

Clearly $\rho_{g}^{[1]}(f)=\rho_{g}(f)$ and $\rho_{\exp z}^{[1]}(f)=\rho_{f}$.

Remark 10. Likewise one can define the generalized relative lower order of $f$ with respect to $g$ denoted by $\lambda_{g}^{[l]}(f)$ as

$$
\lambda_{g}^{[l]}(f)=\liminf _{r \rightarrow \infty} \frac{\log ^{[l]} M_{g}^{-1} M_{f}(r)}{\log r} .
$$

Moreover to compare the relative growth of two entire functions having the same nonzero finite generalized relative order with respect to another entire function, Datta et al. [4] introduced the definition of generalized relative type and generalized relative lower type of an entire function with respect to another entire function, which are as follows.

Definition 11 (see [4]). Assume that $f$ and $g$ are entire functions with $0<\rho_{g}^{[l]}(f)<\infty$. The generalized relative type $\sigma_{g}^{[l]}(f)$ and generalized relative lower type $\bar{\sigma}_{g}^{[l]}(f)$ of $f$ with respect to $g$ are defined as

$$
\begin{aligned}
& \sigma_{g}^{[l]}(f)=\limsup _{r \rightarrow \infty} \frac{\log ^{[l-1]} M_{g}^{-1} M_{f}(r)}{r_{g}^{[l]}(f)}, \\
& \bar{\sigma}_{g}^{[l]}(f)=\liminf _{r \rightarrow \infty} \frac{\log ^{[l-1]} M_{g}^{-1} M_{f}(r)}{r_{g}^{\rho_{g}^{[l]}}(f)} .
\end{aligned}
$$

For $l=2$, Definition 11 reduces to classical definition as established by Roy [15].

Further, to determine the relative growth of two entire functions having the same nonzero finite generalized relative lower order with respect to another entire function, Juneja et al. [10] introduced the concepts of generalized relative weak type and growth indicator of an entire function with respect to another entire function in the following manner.

Definition 12 (see [4]). The generalized relative weak type $\tau_{g}^{[l]}(f)$ and the growth indicator $\bar{\tau}_{g}^{[l]}(f)$ of an entire function $f$ with respect to another entire function $g$ having finite positive generalized relative lower order $\lambda_{g}^{[l]}(f)$ are defined as

$$
\begin{aligned}
& \tau_{g}^{[l]}(f)=\liminf _{r \rightarrow \infty} \frac{\log ^{[l-1]} M_{g}^{-1} M_{f}(r)}{r_{g}^{\lambda_{g}^{[l]}(f)}}, \\
& \begin{aligned}
\bar{\tau}_{g}^{[l]}(f)=\limsup _{r \rightarrow \infty} \frac{\log ^{[l-1]} M_{g}^{-1} M_{f}(r)}{r^{\lambda_{g}^{l l}(f)}}, & \\
& 0<\lambda_{g}^{[l]}(f)<\infty .
\end{aligned}
\end{aligned}
$$


Remark 13. For $l=2$, Definition 12 reduces to the classical definition as established by Datta and Biswas [8].

\section{Lemmas}

In this section we present two lemmas which will be needed in the sequel.

Lemma 14 (see [16]). Let $f$ be an entire function with $0<$ $\lambda_{f}^{[m]} \leq \rho_{f}^{[m]}<\infty$ and let $g$ be entire of regular $(m, p)$-growth, where $p$ and $m$ are positive integers such that $m \geq p$. Then

$$
\begin{aligned}
\rho_{g}^{[p]}(f) & =\frac{\rho_{f}^{[m]}}{\rho_{g}(m, p)}, \\
\lambda_{g}^{[p]}(f) & =\frac{\lambda_{f}^{[p]}}{\lambda_{g}(m, p)} .
\end{aligned}
$$

Lemma 15 (see [16]). Let $f$ be an entire function with $0<$ $\lambda_{f}^{[m]}=\rho_{f}^{[m]}<\infty$ growth and let $g$ be entire with $0 \leq$ $\lambda_{g}(m, p) \leq \rho_{g}(m, p)<\infty$, where $p$ and $m$ are positive integers such that $m \geq p$. Then

$$
\begin{aligned}
& \lambda_{g}^{[p]}(f)=\frac{\rho_{f}^{[m]}}{\rho_{g}(m, p)}, \\
& \rho_{g}^{[p]}(f)=\frac{\lambda_{f}^{[p]}}{\lambda_{g}(m, p)} .
\end{aligned}
$$

\section{Main Results}

In this section we present the main results of the paper.

Theorem 16. Let $f$ be any entire function with $0<\rho_{f}^{[m]}<\infty$ and let $g$ be any entire function with index-pairs $(m, p)$, where $p$ and $m$ are positive integers such that $m \geq p$. Also let $g$ be of regular $(m, p)$-growth. Then

$$
\begin{aligned}
& {\left[\frac{\bar{\sigma}_{f}^{[m]}}{\sigma_{g}(m, p)}\right]^{1 / \rho_{g}(m, p)} \leq \bar{\sigma}_{g}^{[p]}(f)} \\
& \leq \min \left\{\left[\frac{\bar{\sigma}_{f}^{[m]}}{\bar{\sigma}_{g}(m, p)}\right]^{1 / \rho_{g}(m, p)},\right. \\
& \left.\left[\frac{\sigma_{f}^{[m]}}{\sigma_{g}(m, p)}\right]^{1 / \rho_{g}(m, p)}\right\} \\
& \leq \max \left\{\left[\frac{\bar{\sigma}_{f}^{[m]}}{\bar{\sigma}_{g}(m, p)}\right]^{1 / \rho_{g}(m, p)}\right.
\end{aligned}
$$

$$
\begin{aligned}
& \left.\left[\frac{\sigma_{f}^{[m]}}{\sigma_{g}(m, p)}\right]^{1 / \rho_{g}(m, p)}\right\} \leq \\
& \leq\left[\frac{\sigma_{f}^{[m]}}{\bar{\sigma}_{g}(m, p)}\right]^{1 / \rho_{g}(m, p)} .
\end{aligned}
$$

Proof. Fix $\epsilon>0$. From the definitions of $\sigma_{f}^{[m]}$ and $\bar{\sigma}_{f}^{[m]}$ we have for all sufficiently large values of $r$ that

$$
\begin{aligned}
& M_{f}(r) \leq \exp ^{[m-1]}\left\{\left(\sigma_{f}^{[m]}+\varepsilon\right) r^{[m]}\right\}, \\
& M_{f}(r) \geq \exp ^{[m-1]}\left\{\left(\bar{\sigma}_{f}^{[m]}-\varepsilon\right) r^{\rho_{f}^{[m]}}\right\}
\end{aligned}
$$

and also for a sequence of values of $r$ tending to infinity we get that

$$
\begin{aligned}
& M_{f}(r) \geq \exp ^{[m-1]}\left\{\left(\sigma_{f}^{[m]}-\varepsilon\right) r^{\rho_{f}^{[m]}}\right\}, \\
& M_{f}(r) \leq \exp ^{[m-1]}\left\{\left(\bar{\sigma}_{f}^{[m]}+\varepsilon\right) r^{\rho_{f}^{[m]}}\right\} .
\end{aligned}
$$

Similarly, from the definitions of $\sigma_{g}(m, p)$ and $\bar{\sigma}_{g}(m, p)$, it follows for all sufficiently large values of $r$ that

$$
\begin{gathered}
M_{g}(r) \leq \exp ^{[m-1]}\left\{\left(\sigma_{g}(m, p)+\varepsilon\right)\left(\log ^{[p-1]} r\right)^{\rho_{g}(m, p)}\right\} \\
\text { i.e., } r \leq M_{g}^{-1}\left[\operatorname { e x p } ^ { [ m - 1 ] } \left\{\left(\sigma_{g}(m, p)+\varepsilon\right)\right.\right. \\
\left.\left.\cdot\left(\log ^{[p-1]} r\right)^{\rho_{g}^{(m, p)}}\right\}\right] \\
\text { i.e., } M_{g}^{-1}(r) \geq \exp ^{[p-1]}\left[\left(\frac{\log ^{[m-1]} r}{\left(\sigma_{g}(m, p)+\varepsilon\right)}\right)^{1 / \rho_{g}(m, p)}\right], \\
M_{g}(r) \geq \exp ^{[m-1]}\left\{\left(\bar{\sigma}_{g}(m, p)-\varepsilon\right)\left(\log { }^{[p-1]} r\right)^{\rho_{g}(m, p)}\right\} \\
\text { i.e., } r \geq M_{g}^{-1}\left[\operatorname { e x p } ^ { [ m - 1 ] } \left\{\left(\bar{\sigma}_{g}(m, p)-\varepsilon\right)\right.\right. \\
\left.\left..\left(\log ^{[p-1]} r\right)^{\rho_{g}(m, p)}\right\}\right] \\
\text { i.e., } M_{g}^{-1}(r) \leq \exp ^{[p-1]}\left[\left(\frac{\log { }^{[m-1]} r}{\left(\bar{\sigma}_{g}(m, p)-\varepsilon\right)}\right)^{1 / \rho_{g}(m, p)}\right],
\end{gathered}
$$


and for a sequence of values of $r$ tending to infinity we obtain that

$$
\begin{aligned}
& M_{g}(r) \geq \exp ^{[m-1]}\left\{\left(\sigma_{g}(m, p)-\varepsilon\right)\left(\log ^{[p-1]} r\right)^{\rho_{g}(m, p)}\right\} \\
& \text { i.e., } r \geq M_{g}^{-1}\left[\operatorname { e x p } ^ { [ m - 1 ] } \left\{\left(\sigma_{g}(m, p)-\varepsilon\right)\right.\right. \\
& \left.\left.\cdot\left(\log ^{[p-1]} r\right)^{\rho_{g}(m, p)}\right\}\right] \\
& \text { i.e., } M_{g}^{-1}(r) \leq \exp ^{[p-1]}\left[\left(\frac{\log ^{[m-1]} r}{\left(\sigma_{g}(m, p)-\varepsilon\right)}\right)^{1 / \rho_{g}(m, p)}\right],
\end{aligned}
$$

$$
\begin{aligned}
& M_{g}(r) \leq \exp ^{[m-1]}\left\{\left(\bar{\sigma}_{g}(m, p)+\varepsilon\right)\left(\log ^{[p-1]} r\right)^{\rho_{g}(m, p)}\right\} \\
& \text { i.e., } r \leq M_{g}^{-1}\left[\operatorname { e x p } ^ { [ m - 1 ] } \left\{\left(\bar{\sigma}_{g}(m, p)+\varepsilon\right)\right.\right. \\
& \left.\left.\cdot\left(\log ^{[p-1]} r\right)^{\rho_{g}(m, p)}\right\}\right] \\
& \text { i.e., } M_{g}^{-1}(r) \geq \exp ^{[p-1]}\left[\left(\frac{\log ^{[m-1]} r}{\left(\bar{\sigma}_{g}(m, p)-\varepsilon\right)}\right)^{1 / \rho_{g}(m, p)}\right] .
\end{aligned}
$$

Now, from (20) and in view of (22), we get for a sequence of values of $r$ tending to infinity that

$$
\begin{aligned}
\log ^{[p-1]} M_{g}^{-1} M_{f}(r) \geq \log ^{[p-1]} M_{g}^{-1}\left[\exp ^{[m-1]}\left\{\left(\sigma_{f}^{[m]}-\varepsilon\right) r^{\rho_{f}^{[m]}}\right\}\right] \\
\text { i.e., } \log ^{[p-1]} M_{g}^{-1} M_{f}(r) \geq \log ^{[p-1]} \exp ^{[p-1]}\left[\left(\frac{\log ^{[m-1]} \exp ^{[m-1]}\left\{\left(\sigma_{f}^{[m]}-\varepsilon\right) r^{\rho_{f}^{[m]}}\right\}}{\left(\sigma_{g}(m, p)+\varepsilon\right)}\right)^{1 / \rho_{g}(m, p)}\right] \\
\text { i.e., } \left.\log ^{[p-1]} M_{g}^{-1} M_{f}(r) \geq\left[\frac{\left(\sigma_{f}^{[m]}-\varepsilon\right)}{\left(\sigma_{g}(m, p)+\varepsilon\right)}\right]^{1 / \rho_{g}(m, p)} \cdot r_{f}^{\rho_{f}^{[m]} / \rho_{g}(m, p)}\right] \\
\text { i.e., } \frac{\log ^{[p-1]} M_{g}^{-1} M_{f}(r)}{r^{[m]} / \rho_{g}(m, p)} \geq\left[\frac{\left(\sigma_{f}^{[m]}-\varepsilon\right)}{\left(\sigma_{g}(m, p)+\varepsilon\right)}\right]^{1 / \rho_{g}(m, p)} .
\end{aligned}
$$

As $\varepsilon(>0)$ is arbitrary, in view of Lemma 14 , it follows that

$$
\begin{aligned}
\limsup _{r \rightarrow \infty} \frac{\log ^{[p-1]} M_{g}^{-1} M_{f}(r)}{r^{\rho_{g}^{[p]}(f)}} & \geq\left[\frac{\sigma_{f}^{[m]}}{\sigma_{g}(m, p)}\right]^{1 / \rho_{g}(m, p)} \\
\text { i.e., } \sigma_{g}^{[p]}(f) & \geq\left[\frac{\sigma_{f}^{[m]}}{\sigma_{g}(m, p)}\right]^{1 / \rho_{g}(m, p)} .
\end{aligned}
$$

Analogously from (19) and in view of (25) it follows for a sequence of values of $r$ tending to infinity that

$$
\begin{aligned}
\log ^{[p-1]} M_{g}^{-1} M_{f}(r) \geq \log ^{[p-1]} M_{g}^{-1}\left[\exp ^{[m-1]}\left\{\left(\bar{\sigma}_{f}^{[m]}-\varepsilon\right) r_{f}^{\rho_{f}^{[m]}}\right\}\right] \\
\text { i.e., } \log ^{[p-1]} M_{g}^{-1} M_{f}(r) \geq \log ^{[p-1]} \exp ^{[p-1]}\left[\left(\frac{\log ^{[m-1]} \exp ^{[m-1]}\left\{\left(\bar{\sigma}_{f}^{[m]}-\varepsilon\right) r^{\rho_{f}^{[m]}}\right\}}{\left(\bar{\sigma}_{g}(m, p)+\varepsilon\right)}\right)^{1 / \rho_{g}(m, p)}\right] \\
\text { i.e., } \log ^{[p-1]} M_{g}^{-1} M_{f}(r) \geq\left[\frac{\left(\bar{\sigma}_{f}^{[m]}-\varepsilon\right)}{\left(\bar{\sigma}_{g}(m, p)+\varepsilon\right)}\right]^{1 / \rho_{g}(m, p)} \cdot r_{f}^{[m] / \rho_{g}(m, p)} \\
\text { i.e., } \frac{\log ^{[p-1]} M_{g}^{-1} M_{f}(r)}{r_{f}^{\left.\rho_{f}\right] / \rho_{g}(m, p)}} \geq\left[\frac{\left(\bar{\sigma}_{f}^{[m]}-\varepsilon\right)}{\left(\bar{\sigma}_{g}(m, p)+\varepsilon\right)}\right]^{1 / \rho_{g}(m, p)} .
\end{aligned}
$$


Since $\varepsilon(>0)$ is arbitrary, we get from the above and Lemma 14 that

$$
\begin{array}{r}
\limsup _{r \rightarrow \infty} \frac{\log ^{[p-1]} M_{g}^{-1} M_{f}(r)}{r^{\rho_{g}^{[p]}(f)}} \geq\left[\frac{\bar{\sigma}_{f}^{[m]}}{\bar{\sigma}_{g}(m, p)}\right]^{1 / \rho_{g}(m, p)} \\
\text { i.e., } \sigma_{g}^{[p]}(f) \geq\left[\frac{\bar{\sigma}_{f}^{[m]}}{\bar{\sigma}_{g}(m, p)}\right]^{1 / \rho_{g}(m, p)} .
\end{array}
$$

Again in view of (23) we have from (18), for all sufficiently large values of $r$, that

$$
\begin{aligned}
\log ^{[p-1]} M_{g}^{-1} M_{f}(r) \leq \log ^{[p-1]} M_{g}^{-1}\left[\exp ^{[m-1]}\left\{\left(\sigma_{f}^{[m]}+\varepsilon\right) r_{f}^{\rho_{f}^{[m]}}\right\}\right] \\
\text { i.e., } \log ^{[p-1]} M_{g}^{-1} M_{f}(r) \leq \log ^{[p-1]} \exp ^{[p-1]}\left[\left(\frac{\log ^{[m-1]} \exp ^{[m-1]}\left\{\left(\sigma_{f}^{[m]}+\varepsilon\right) r^{[m]}\right\}}{\left(\bar{\sigma}_{g}(m, p)-\varepsilon\right)}\right)^{1 / \rho_{g}(m, p)}\right] \\
\text { i.e., } \left.\log ^{[p-1]} M_{g}^{-1} M_{f}(r) \leq\left[\frac{\left(\sigma_{f}^{[m]}+\varepsilon\right)}{\left(\bar{\sigma}_{g}(m, p)-\varepsilon\right)}\right]^{1 / \rho_{g}(m, p)} \cdot r_{f}^{\rho_{f}^{[m]} / \rho_{g}(m, p)}\right] \\
\text { i.e., } \frac{\log ^{[p-1]} M_{g}^{-1} M_{f}(r)}{r^{\rho_{f}^{[m]} / \rho_{g}(m, p)}} \leq\left[\frac{\left(\sigma_{f}^{[m]}+\varepsilon\right)}{\left(\bar{\sigma}_{g}(m, p)-\varepsilon\right)}\right]^{1 / \rho_{g}(m, p)} \cdot
\end{aligned}
$$

Since $\varepsilon(>0)$ is arbitrary, we obtain in view of Lemma 14 that

$$
\begin{array}{r}
\limsup _{r \rightarrow \infty} \frac{\log ^{[p-1]} M_{g}^{-1} M_{f}(r)}{r_{g}^{[p]}(f)} \leq\left[\frac{\sigma_{f}^{[m]}}{\bar{\sigma}_{g}(m, p)}\right]^{1 / \rho_{g}(m, p)} \\
\text { i.e., } \sigma_{g}^{[p]}(f) \leq\left[\frac{\sigma_{f}^{[m]}}{\bar{\sigma}_{g}(m, p)}\right]^{1 / \rho_{g}(m, p)} .
\end{array}
$$

Again, from (19) and in view of (22), we get for all sufficiently large values of $r$ that

$$
\begin{gathered}
\log ^{[p-1]} M_{g}^{-1} M_{f}(r) \geq \log ^{[p-1]} M_{g}^{-1}\left[\exp ^{[m-1]}\left\{\left(\bar{\sigma}_{f}^{[m]}-\varepsilon\right) r_{f}^{\rho_{f}^{[m]}}\right\}\right] \\
\text { i.e., } \log ^{[p-1]} M_{g}^{-1} M_{f}(r) \geq \log ^{[p-1]} \exp ^{[p-1]}\left[\left(\frac{\log ^{[m-1]} \exp ^{[m-1]}\left\{\left(\bar{\sigma}_{f}^{[m]}-\varepsilon\right) r_{f}^{[m]}\right\}}{\left(\sigma_{g}(m, p)+\varepsilon\right)}\right)^{1 / \rho_{g}(m, p)}\right] \\
\text { i.e., } \log ^{[p-1]} M_{g}^{-1} M_{f}(r) \geq\left[\frac{\left(\bar{\sigma}_{f}^{[m]}-\varepsilon\right)}{\left(\sigma_{g}(m, p)+\varepsilon\right)}\right]^{1 / \rho_{g}(m, p)} \cdot r_{f}^{\rho_{f}^{[m]} / \rho_{g}(m, p)} \\
\text { i.e., } \frac{\log ^{[p-1]} M_{g}^{-1} M_{f}(r)}{r^{\rho_{f}^{[m]} / \rho_{g}(m, p)}} \geq\left[\frac{\left(\bar{\sigma}_{f}^{[m]}-\varepsilon\right)}{\left(\sigma_{g}(m, p)+\varepsilon\right)}\right]^{1 / \rho_{g}(m, p)} \cdot
\end{gathered}
$$


As $\varepsilon(>0)$ is arbitrary, it follows from the above and Lemma 14 that

$$
\begin{aligned}
\liminf _{r \rightarrow \infty} \frac{\log ^{[p-1]} M_{g}^{-1} M_{f}(r)}{r_{g}^{\rho_{g}^{[p]}(f)}} \geq\left[\frac{\bar{\sigma}_{f}^{[m]}}{\sigma_{g}(m, p)}\right]^{1 / \rho_{g}(m, p)} \\
\text { i.e., } \bar{\sigma}_{g}^{[p]}(f) \geq\left[\frac{\bar{\sigma}_{f}^{[m]}}{\sigma_{g}(m, p)}\right]^{1 / \rho_{g}(m, p)} .
\end{aligned}
$$

Also, in view of (24), we get from (18) that, for a sequence of $r$ tending to infinity,

$$
\begin{gathered}
\log ^{[p-1]} M_{g}^{-1} M_{f}(r) \leq \log ^{[p-1]} M_{g}^{-1}\left[\exp ^{[m-1]}\left\{\left(\sigma_{f}^{[m]}+\varepsilon\right) r_{f}^{\rho_{f}^{[m]}}\right\}\right] \\
\text { i.e., } \log ^{[p-1]} M_{g}^{-1} M_{f}(r) \leq \log ^{[p-1]} \exp ^{[p-1]}\left[\left(\frac{\log ^{[m-1]} \exp ^{[m-1]}\left\{\left(\sigma_{f}^{[m]}+\varepsilon\right) r_{f}^{[m]}\right\}}{\left(\sigma_{g}(m, p)-\varepsilon\right)}\right)^{1 / \rho_{g}(m, p)}\right] \\
\text { i.e., } \log ^{[p-1]} M_{g}^{-1} M_{f}(r) \leq\left[\frac{\left(\sigma_{f}^{[m]}+\varepsilon\right)}{\left(\sigma_{g}(m, p)-\varepsilon\right)}\right]^{1 / \rho_{g}(m, p)} \cdot r^{\rho_{f}^{[m]} / \rho_{g}(m, p)} \\
\text { i.e., } \frac{\log ^{[p-1]} M_{g}^{-1} M_{f}(r)}{r^{\rho_{f}^{[m]} / \rho_{g}(m, p)}} \leq\left[\frac{\left(\sigma_{f}^{[m]}+\varepsilon\right)}{\left(\sigma_{g}(m, p)-\varepsilon\right)}\right]^{1 / \rho_{g}(m, p)} \cdot
\end{gathered}
$$

Since $\varepsilon(>0)$ is arbitrary, we get from Lemma 14 and the above that

$$
\begin{array}{r}
\liminf _{r \rightarrow \infty} \frac{\log ^{[p-1]} M_{g}^{-1} M_{f}(r)}{r_{g}^{\rho_{g}^{[p]}(f)}} \leq\left[\frac{\sigma_{f}^{[m]}}{\sigma_{g}(m, p)}\right]^{1 / \rho_{g}(m, p)} \\
\text { i.e., } \bar{\sigma}_{g}^{[p]}(f) \leq\left[\frac{\sigma_{f}^{[m]}}{\sigma_{g}(m, p)}\right]^{1 / \rho_{g}(m, p)} .
\end{array}
$$

Similarly, from (21) and in view of (23), it follows for a sequence of values of $r$ tending to infinity that

$$
\begin{aligned}
& \log ^{[p-1]} M_{g}^{-1} M_{f}(r) \leq \log ^{[p-1]} M_{g}^{-1}\left[\exp ^{[m-1]}\left\{\left(\bar{\sigma}_{f}^{[m]}+\varepsilon\right) r_{f}^{\rho_{f}^{[m]}}\right\}\right] \\
& \text { i.e., } \log ^{[p-1]} M_{g}^{-1} M_{f}(r) \leq \log ^{[p-1]} \exp ^{[p-1]}\left[\left(\frac{\log ^{[m-1]} \exp ^{[m-1]}\left\{\left(\bar{\sigma}_{f}^{[m]}+\varepsilon\right) r_{f}^{[m]}\right\}}{\left(\bar{\sigma}_{g}(m, p)-\varepsilon\right)}\right)^{1 / \rho_{g}(m, p)}\right] \\
& \text { i.e., } \left.\log ^{[p-1]} M_{g}^{-1} M_{f}(r) \leq\left[\frac{\left(\bar{\sigma}_{f}^{[m]}+\varepsilon\right)}{\left(\bar{\sigma}_{g}(m, p)-\varepsilon\right)}\right]^{1 / \rho_{g}(m, p)} \cdot r_{f}^{\rho_{f}^{[m]} / \rho_{g}(m, p)}\right] \\
& \text { i.e., } \frac{\log ^{[p-1]} M_{g}^{-1} M_{f}(r)}{r^{\rho_{f}^{[m]} / \rho_{g}(m, p)}} \leq\left[\frac{\left(\bar{\sigma}_{f}^{[m]}+\varepsilon\right)}{\left(\bar{\sigma}_{g}(m, p)-\varepsilon\right)}\right]^{1 / \rho_{g}(m, p)} \cdot
\end{aligned}
$$


As $\varepsilon(>0)$ is arbitrary, we obtain from Lemma 14 and the above that

$$
\begin{aligned}
\liminf _{r \rightarrow \infty} \frac{\log ^{[p-1]} M_{g}^{-1} M_{f}(r)}{r^{\rho_{g}^{[p]}(f)}} \leq\left[\frac{\bar{\sigma}_{f}^{[m]}}{\bar{\sigma}_{g}(m, p)}\right]^{1 / \rho_{g}(m, p)} \\
\text { i.e., } \bar{\sigma}_{g}^{[p]}(f) \leq\left[\frac{\bar{\sigma}_{f}^{[m]}}{\bar{\sigma}_{g}(m, p)}\right]^{1 / \rho_{g}(m, p)} .
\end{aligned}
$$

Thus the theorem follows from (27), (29), (31), (33), (35), and (37).

In view of Theorem 16, one can easily verify the following corollaries.

Corollary 17. Let $f$ be an entire function such that $\sigma_{f}^{[\mathrm{m}]}=\bar{\sigma}_{f}^{[\mathrm{m}]}$ and let $g$ be an entire function of regular $(m, p)$-growth, where $p$ and $m$ are all positive integers such that $m \geq p$. Then

$$
\begin{aligned}
& \sigma_{g}^{[p]}(f)=\left[\frac{\sigma_{f}^{[m]}}{\bar{\sigma}_{g}(m, p)}\right]^{1 / \rho_{g}(m, p)}, \\
& \bar{\sigma}_{g}^{[p]}(f)=\left[\frac{\sigma_{f}^{[m]}}{\sigma_{g}(m, p)}\right]^{1 / \rho_{g}(m, p)} .
\end{aligned}
$$

Corollary 18. Let $f$ be an entire function with $0<\rho_{f}^{[m]}<\infty$ and let $g$ be entire function of perfectly regular $(m, p)$-growth, where $p$ and $m$ are positive integers with $m \geq p$. Then

$$
\begin{aligned}
& \sigma_{g}^{[p]}(f)=\left[\frac{\sigma_{f}^{[m]}}{\sigma_{g}(m, p)}\right]^{1 / \rho_{g}(m, p)}, \\
& \bar{\sigma}_{g}^{[p]}(f)=\left[\frac{\bar{\sigma}_{f}^{[m]}}{\sigma_{g}(m, p)}\right]^{1 / \rho_{g}(m, p)} .
\end{aligned}
$$

In addition, if $\sigma_{f}^{[m]}=\bar{\sigma}_{f}^{[m]}$, then

$$
\sigma_{g}^{[p]}(f)=\bar{\sigma}_{g}^{[p]}(f)=\left[\frac{\sigma_{f}^{[m]}}{\sigma_{g}(m, p)}\right]^{1 / \rho_{g}(m, p)} .
$$

Corollary 19. Let $f$ be an entire function with $0<\rho_{f}^{[m]}<\infty$. Then, for any entire function $g$,

(i) $\bar{\sigma}_{g}^{[p]}(f)=\infty$ when $\sigma_{g}(m, p)=0$,

(ii) $\sigma_{g}^{[p]}(f)=\infty$ when $\bar{\sigma}_{g}(m, p)=0$,

(iii) $\bar{\sigma}_{g}^{[p]}(f)=0$ when $\sigma_{g}(m, p)=\infty$,

(iv) $\sigma_{g}^{[p]}(f)=\infty$ when $\bar{\sigma}_{g}(m, p)=\infty$,

where $p$ is any positive integer with $m \geq p$ and $g$ is of regular $(m, p)$-growth.
Corollary 20. Let $g$ be an entire function with regular $(m, p)$-growth where $m, p$ are positive integers with $m \geq p$. Then, for any entire function $f$ with $0<\rho_{f}^{[m]}<\infty$, one has

(i) $\sigma_{g}^{[p]}(f)=0$ when $\sigma_{f}^{[m]}=0$,

(ii) $\bar{\sigma}_{g}^{[p]}(f)=0$ when $\bar{\sigma}_{f}^{[m]}=0$,

(iii) $\sigma_{g}^{[p]}(f)=\infty$ when $\sigma_{f}^{[m]}=\infty$,

(iv) $\bar{\sigma}_{g}^{[p]}(f)=\infty$ when $\bar{\sigma}_{f}^{[m]}=\infty$.

In line with Theorem 16 and with the help of Lemma 15, one can prove the following theorem, and therefore its proof is omitted.

Theorem 21. Let $f$ be any entire function with $0<\lambda_{f}^{[m]}=$ $\rho_{f}^{[m]}<\infty$ and let $g$ be any entire function with index-pairs $(m, p)$, where $p$ and $m$ are positive integers such that $m \geq p$. Then

$$
\begin{aligned}
& {\left[\frac{\bar{\sigma}_{f}^{[m]}}{\sigma_{g}(m, p)}\right]^{1 / \rho_{g}(m, p)} \leq \tau_{g}^{[p]}(f)} \\
& \leq \min \left\{\left[\frac{\bar{\sigma}_{f}^{[m]}}{\bar{\sigma}_{g}(m, p)}\right]^{1 / \rho_{g}(m, p)},\right. \\
& \left.\left[\frac{\sigma_{f}^{[m]}}{\sigma_{g}(m, p)}\right]^{1 / \rho_{g}(m, p)}\right\} \\
& \leq \max \left\{\left[\frac{\bar{\sigma}_{f}^{[m]}}{\bar{\sigma}_{g}(m, p)}\right]^{1 / \rho_{g}(m, p)},\right. \\
& \left.\leq\left[\frac{\sigma_{f}^{[m]}}{\bar{\sigma}_{g}(m, p)}\right]^{1 / \rho_{g}(m, p)}\right]^{1 / \rho_{g}(m, p)} \\
& {\left[\begin{array}{c}
\sigma_{g}(m, p) \\
{[p]}
\end{array}\right]}
\end{aligned}
$$

In view of Theorem 21, one can easily derive the following corollaries.

Corollary 22. Let $f$ be an entire function with $0<\lambda_{f}^{[m]}=$ $\rho_{f}^{[m]}<\infty$ and $\sigma_{f}^{[m]}=\bar{\sigma}_{f}^{[m]}$ and let $g$ be an entire function with 
index-pairs $(m, p)$, where $p$ and $m$ are positive integers such that $m \geq p$. Then

$$
\begin{aligned}
& \bar{\tau}_{g}^{[p]}(f)=\left[\frac{\sigma_{f}^{[m]}}{\bar{\sigma}_{g}(m, p)}\right]^{1 / \rho_{g}(m, p)}, \\
& \tau_{g}^{[p]}(f)=\left[\frac{\sigma_{f}^{[m]}}{\sigma_{g}(m, p)}\right]^{1 / \rho_{g}(m, p)} .
\end{aligned}
$$

Corollary 23. Let $f$ be an entire function with $0<\lambda_{f}^{[m]}=$ $\rho_{f}^{[m]}<\infty$ and let $g$ be an entire function with $\sigma_{g}(m, p)=$ $\bar{\sigma}_{g}(m, p)$. Then

$$
\begin{gathered}
\bar{\tau}_{g}^{[p]}(f)=\left[\frac{\sigma_{f}^{[m]}}{\sigma_{g}(m, p)}\right]^{1 / \rho_{g}(m, p)}, \\
\tau_{g}^{p}(f)=\left[\frac{\bar{\sigma}_{f}^{[m]}}{\sigma_{g}(m, p)}\right]^{1 / \rho_{g}(m, p)} .
\end{gathered}
$$

In addition, if $\sigma_{f}^{[m]}=\bar{\sigma}_{f}^{[m]}$, then

$$
\bar{\tau}_{g}^{[p]}(f)=\tau_{g}^{[p]}(f)=\left[\frac{\sigma_{f}^{[m]}}{\sigma_{g}(m, p)}\right]^{1 / \rho_{g}(m, p)} .
$$

Corollary 24. Let $g$ be an entire function with index-pairs $(m, p)$, where $p$ and $m$ are positive integers such that $m \geq p$. Then, for any entire function $f$ with $0<\lambda_{f}^{[m]}=\rho_{f}^{[m]}<\infty$, one has

(i) $\tau_{g}^{[p]}(f)=\infty$ when $\sigma_{g}(m, p)=0$,

(ii) $\bar{\tau}_{g}^{[p]}(f)=\infty$ when $\bar{\sigma}_{g}(m, p)=0$,

(iii) $\tau_{g}^{p}(f)=0$ when $\sigma_{g}(m, p)=\infty$,

(iv) $\bar{\tau}_{g}^{[p]}(f)=\infty$ when $\bar{\sigma}_{g}(m, p)=\infty$.

Corollary 25. Let $f$ be an entire function with $0<\lambda_{f}^{[m]}=$ $\rho_{f}^{[m]}<\infty$. Then for any entire function $g$ with index-pairs $(m, p)$, where $p$ and $m$ are positive integers such that $m \geq p$, one has

(i) $\bar{\tau}_{g}^{[p]}(f)=0$ when $\sigma_{f}^{[m]}=0$,

(ii) $\tau_{g}^{[p]}(f)=0$ when $\bar{\sigma}_{f}^{[m]}=0$,

(iii) $\bar{\tau}_{g}^{[p]}(f)=\infty$ when $\sigma_{f}^{[m]}=\infty$,

(iv) $\tau_{g}^{[p]}(f)=\infty$ when $\bar{\sigma}_{f}^{[m]}=\infty$.

Similarly, in line with Theorems 16 and 21 and with the help of Lemmas 14 and 15, one may easily prove the following two theorems, and therefore their proofs are omitted.
Theorem 26. Let $f$ be any entire function with $0<\lambda_{f}^{[m]}<\infty$ and let $g$ be any entire function with index-pairs $(m, p)$, where $p$ and $m$ are positive integers such that $m \geq p$. Also let $g$ be of regular $(m, p)$-growth. Then

$$
\begin{aligned}
& {\left[\frac{\tau_{f}^{[m]}}{\bar{\tau}_{g}(m, p)}\right]^{1 / \lambda_{g}(m, p)} \leq \tau_{g}^{[p]}(f)} \\
& \leq \min \left\{\left[\frac{\tau_{f}^{[m]}}{\tau_{g}(m, p)}\right]^{1 / \lambda_{g}(m, p)},\right. \\
& \left.\left[\frac{\bar{\tau}_{f}^{[m]}}{\bar{\tau}_{g}(m, p)}\right]^{1 / \lambda_{g}(m, p)}\right\} \\
& \leq \max \left\{\left[\frac{\tau_{f}^{[m]}}{\tau_{g}(m, p)}\right]^{1 / \lambda_{g}(m, p)},\right. \\
& \left.\left[\frac{\bar{\tau}_{f}^{m}}{\bar{\tau}_{g}(m, p)}\right]^{1 / \lambda_{g}(m, p)}\right\} \leq \bar{\tau}_{g}^{[p]}(f) \\
& \leq\left[\frac{\bar{\tau}_{f}^{[m]}}{\tau_{g}(m, p)}\right]^{1 / \lambda_{g}(m, p)} .
\end{aligned}
$$

In view of Theorem 26, the following corollaries may also be obtained.

Corollary 27. Let $f$ be an entire function such that $\tau_{f}^{[m]}=\bar{\tau}_{f}^{[m]}$ and let $g$ be an entire function of regular $(m, p)$-growth, where $p$ and $m$ are positive integers such that $m \geq p$. Then

$$
\begin{aligned}
& \bar{\tau}_{g}^{[p]}(f)=\left[\frac{\tau_{f}^{[m]}}{\tau_{g}(m, p)}\right]^{1 / \lambda_{g}(m, p)}, \\
& \tau_{g}^{[p]}(f)=\left[\frac{\tau_{f}^{[m]}}{\bar{\tau}_{g}(m, p)}\right]^{1 / \lambda_{g}(m, p)} .
\end{aligned}
$$

Corollary 28. Let $f$ be an entire function with $0<\lambda_{f}^{[m]}<\infty$ and let $g$ be an entire function of regular $(m, p)$-growth, where $p$ and $m$ are positive integers such that $m \geq p$ with $\tau_{g}(m, p)=$ $\bar{\tau}_{g}(m, p)$. Then

$$
\begin{aligned}
& \bar{\tau}_{g}^{[p]}(f)=\left[\frac{\bar{\tau}_{f}^{[m]}}{\bar{\tau}_{g}(m, p)}\right]^{1 / \lambda_{g}(m, p)}, \\
& \tau_{g}^{[p]}(f)=\left[\frac{\tau_{f}^{[m]}}{\bar{\tau}_{g}(m, p)}\right]^{1 / \lambda_{g}(m, p)} .
\end{aligned}
$$


In addition, if $\tau_{f}^{[m]}=\bar{\tau}_{f}^{[m]}$, then

$$
\tau_{g}^{[p]}(f)=\bar{\tau}_{g}^{[p]}(f)=\left[\frac{\bar{\tau}_{f}^{[m]}}{\bar{\tau}_{g}(m, p)}\right]^{1 / \lambda_{g}(m, p)} .
$$

Corollary 29. Let $f$ be an entire function with $0<\lambda_{f}^{[m]}<\infty$ and let $g$ be an entire function of regular $(m, p)$-growth, where $p$ and $m$ are positive integers such that $m \geq p$. Then

(i) $\tau_{g}^{[p]}(f)=\infty$ when $\bar{\tau}_{g}(m, p)=0$,

(ii) $\bar{\tau}_{g}^{[p]}(f)=\infty$ when $\tau_{g}(m, p)=0$,

(iii) $\tau_{g}^{[p]}(f)=0$ when $\bar{\tau}_{g}(m, p)=\infty$,

(iv) $\bar{\tau}_{g}^{[p]}(f)=\infty$ when $\tau_{g}(m, p)=\infty$.

Corollary 30. Let $g$ be an entire function with regular $(m, p)$-growth, where $p$ and $m$ are all positive integers such that $m \geq p$. Then, for any entire function $f$ with $0<\lambda_{f}^{[m]}<\infty$, one has

(i) $\bar{\tau}_{g}^{[p]}(f)=0$ when $\bar{\tau}_{f}^{[m]}=0$,

(ii) $\tau_{g}^{[p]}(f)=0$ when $\tau_{f}^{[m]}=0$,

(iii) $\bar{\tau}_{g}^{[p]}(f)=\infty$ when $\bar{\tau}_{f}^{[m]}=\infty$,

(iv) $\tau_{g}^{[p]}(f)=\infty$ when $\tau_{f}^{[m]}=\infty$.

Theorem 31. Let $f$ be any entire function with $0<\lambda_{f}^{[m]}=$ $\rho_{f}^{[m]}<\infty$ and let $g$ be any entire function with index-pairs $(m, p)$, where $p$ and $m$ are positive integers such that $m \geq p$. Then

$$
\begin{aligned}
& {\left[\frac{\tau_{f}^{[m]}}{\bar{\tau}_{g}(m, p)}\right]^{1 / \lambda_{g}(m, p)} \leq \bar{\sigma}_{g}^{[p]}(f)} \\
& \leq \min \left\{\left[\frac{\tau_{f}^{[m]}}{\tau_{g}(m, p)}\right]^{1 / \lambda_{g}},\left[\frac{\bar{\tau}_{f}^{[m]}}{\bar{\tau}_{g}(m, p)}\right]^{1 / \lambda_{g}(m, p)}\right\} \\
& \leq \max \left\{\left[\frac{\tau_{f}^{[m]}}{\tau_{g}(m, p)}\right]^{1 / \lambda_{g}},\left[\frac{\bar{\tau}_{f}^{[m]}}{\bar{\tau}_{g}(m, p)}\right]^{1 / \lambda_{g}(m, p)}\right\} \\
& \left.\leq \sigma_{g}^{[p]}(f) \leq\left[\frac{\bar{\tau}_{f}^{[m]}}{\tau_{g}(m, p)}\right]^{1 / \lambda_{g}(m, p)}\right\}
\end{aligned}
$$

From Theorem 31 the following corollaries are immediate.

Corollary 32. Let $f$ be an entire function with $0<\lambda_{f}^{[m]}=$ $\rho_{f}^{[m]}<\infty$ and $\bar{\tau}_{f}^{[m]}=\tau_{f}^{[m]}$ and let $g$ be an entire function with index-pairs $(m, p)$, where $p$ and $m$ are positive integers such that $m \geq p$ and $\lambda_{g}(m, p)>0$. Then

$$
\begin{aligned}
& \sigma_{g}^{[p]}(f)=\left[\frac{\bar{\tau}_{f}^{[m]}}{\tau_{g}(m, p)}\right]^{1 / \lambda_{g}(m, p)}, \\
& \bar{\sigma}_{g}^{[p]}(f)=\left[\frac{\bar{\tau}_{f}^{[m]}}{\bar{\tau}_{g}(m, p)}\right]^{1 / \lambda_{g}(m, p)} .
\end{aligned}
$$

Corollary 33. Let $f$ be an entire function with regular growth and let $g$ be an entire function with $\tau_{g}(m, p)=\bar{\tau}_{g}(m, p)$, where $p$ and $m$ are positive integers such that $m \geq p$. Then

$$
\begin{aligned}
& \sigma_{g}^{[p]}(f)=\left[\frac{\bar{\tau}_{f}^{[m]}}{\bar{\tau}_{g}(m, p)}\right]^{1 / \lambda_{g}(m, p)}, \\
& \bar{\sigma}_{g}^{[p]}(f)=\left[\frac{\tau_{f}^{[m]}}{\bar{\tau}_{g}(m, p)}\right]^{1 / \lambda_{g}(m, p)} .
\end{aligned}
$$

In addition, if $\tau_{f}^{[m]}=\bar{\tau}_{f}^{[m]}$, then

$$
\sigma_{g}^{[p]}(f)=\bar{\sigma}_{g}^{[p]}(f)=\left[\frac{\bar{\tau}_{f}^{[m]}}{\bar{\tau}_{g}(m, p)}\right]^{1 / \lambda_{g}(m, p)} .
$$

Corollary 34. Let $g$ be an entire function with index-pairs $(m, p)$, where $p$ and $m$ are positive integers such that $m \geq p$ and $\lambda_{g}(m, p)>0$. Then, for any entire function $f$ with $0<$ $\lambda_{f}^{[m]}=\rho_{f}^{[m]}<\infty$, one has

(i) $\bar{\sigma}_{g}^{[p]}(f)=\infty$ when $\bar{\tau}_{g}(m, p)=0$,

(ii) $\sigma_{g}^{[p]}(f)=\infty$ when $\tau_{g}(m, p)=0$,

(iii) $\bar{\sigma}_{g}^{[p]}(f)=0$ when $\bar{\tau}_{g}(m, p)=\infty$,

(iv) $\sigma_{g}^{[p]}(f)=\infty$ when $\tau_{g}(m, p)=\infty$.

Corollary 35. Let $f$ be an entire function with $0<\lambda_{f}^{[m]}=$ $\rho_{f}^{[m]}<\infty$. Then, for any entire function $g$ with index-pairs $(m, p)$, where $p$ and $m$ are positive integers such that $m \geq p$, one has

(i) $\sigma_{g}^{[p]}(f)=0$ when $\bar{\tau}_{f}^{[m]}=0$,

(ii) $\bar{\sigma}_{g}^{[p]}(f)=0$ when $\tau_{f}^{[m]}=0$,

(iii) $\sigma_{g}^{[p]}(f)=\infty$ when $\bar{\tau}_{f}^{[m]}=\infty$,

(iv) $\bar{\sigma}_{g}^{[p]}(f)=\infty$ when $\tau_{f}^{[m]}=\infty$.

\section{Competing Interests}

The authors declare that there are no competing interests regarding the publication of this paper. 


\section{References}

[1] L. Bernal, Crecimiento relativo de funciones enteras. Contribución al estudio de lasfunciones enteras con índice exponencial finito [Ph.D. thesis], University of Seville, Seville, Spain, 1984.

[2] L. Bernal, "Orden relativeo de crecimiento de funciones enteras," Collectanea Mathematica, vol. 39, pp. 209-229, 1988.

[3] B. K. Lahiri and D. Banerjee, "Generalised relative order of entire functions," Proceedings of the National Academy of Sciences, India-Section A, vol. 72, no. 4, pp. 351-371, 2002.

[4] S. K. Datta, T. Biswas, and C. Ghosh, "Growth analysis of entire functions concerning generalized relative type and generalized relative weak type," Facta Universitatis Series: Mathematics and Informatics, vol. 30, no. 3, pp. 295-324, 2015.

[5] S. K. Datta and T. Biswas, "Growth of entire functions based on relative order," International Journal of Pure and Applied Mathematics, vol. 51, no. 1, pp. 49-58, 2009.

[6] S. K. Datta, T. Biswas, and R. Biswas, "On relative order based on growth estimates of entire functions," International Journal of Mathematical Sciences \& Industrial Applications, vol. 7, no. 2, pp. 59-67, 2013.

[7] S. K. Datta, T. Biswas, and R. Biswas, "Comparative growth properties of composite entire functions in the light of their relative order," The Mathematics Student, vol. 82, no. 1-4, pp. 1-8, 2013.

[8] S. K. Datta and A. Biswas, "On relative type of entire and meromorphic functions," Advances in Applied Mathematical Analysis, vol. 8, no. 2, pp. 63-75, 2013.

[9] G. Valiron, Lectures on the General Theory of Integral Functions, Chelsea Publishing Company, 1949.

[10] O. P. Juneja, G. P. Kapoor, and S. K. Bajpai, "On the (p; q)-order and lower (p; q)-order of an entire function," Journal für die Reine und Angewandte Mathematik, vol. 282, pp. 53-67, 1976.

[11] D. Sato, "On the rate of growth of entire functions of fast growth," Bulletin of the American Mathematical Society, vol. 69, pp. 411-414, 1963.

[12] O. P. Juneja, G. P. Kapoor, and S. K. Bajpai, "On the ( $p$; q)-type and lower $(p ; q)$-type of an entire function," Journal Für die Reine und Angewandte Mathematik, vol. 290, pp. 180-189, 1977.

[13] S. K. Datta and A. Jha, "On the weak type of meromorphic functions," International Mathematical Forum, vol. 4, no. 12, pp. 569-579, 2009.

[14] E. C. Titchmarsh, The Theory of Functions, Oxford University Press, Oxford, UK, 2nd edition, 1968.

[15] C. Roy, Some properties of entire functions in one and several complex variables [Ph.D. thesis], University of Calcutta, 2009.

[16] L. M. Sánchez Ruiz, S. K. Datta, T. Biswas, and G. K. Mondal, "On the $(p, q)$ th relative order oriented growth properties of entire functions," Abstract and Applied Analysis, vol. 2014, Article ID 826137, 8 pages, 2014. 


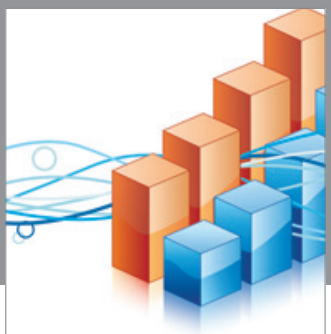

Advances in

Operations Research

vatem alat4

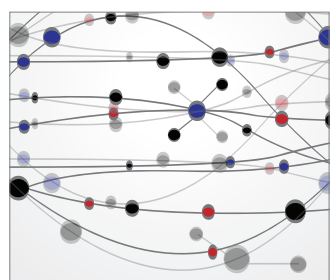

\section{The Scientific} World Journal
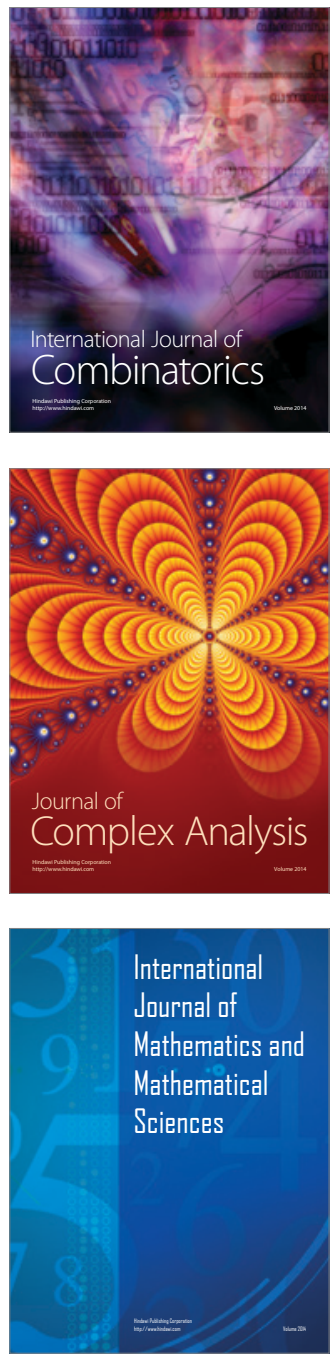
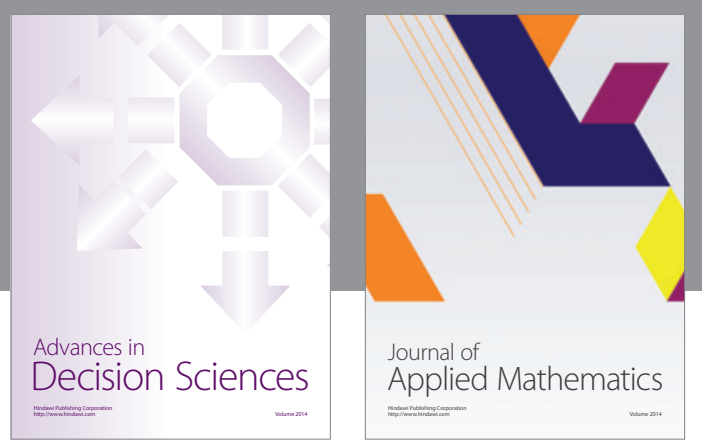

Algebra

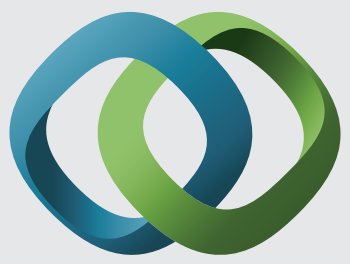

\section{Hindawi}

Submit your manuscripts at

http://www.hindawi.com
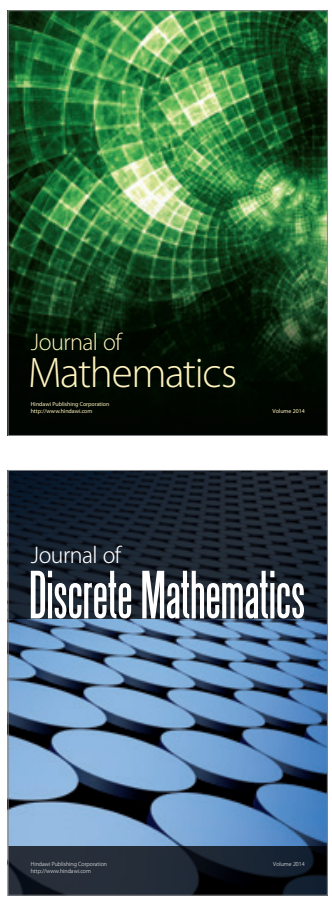

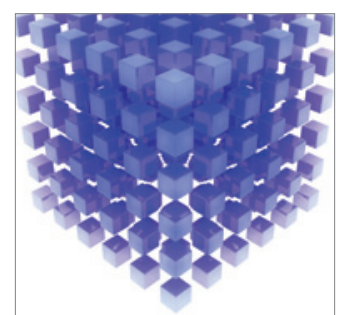

Mathematical Problems in Engineering
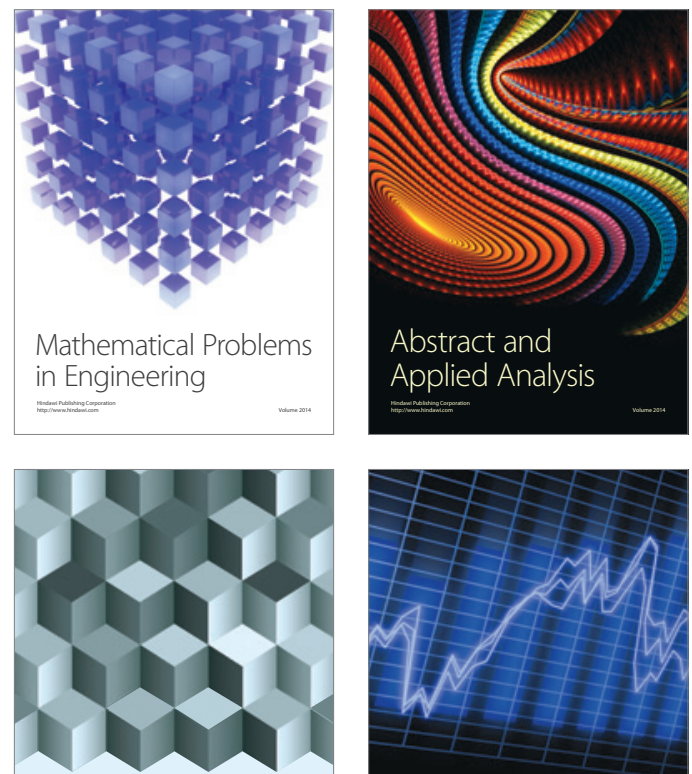

Journal of

Function Spaces

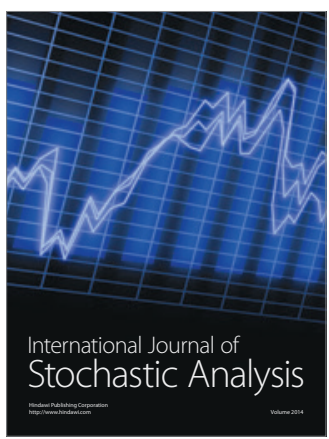

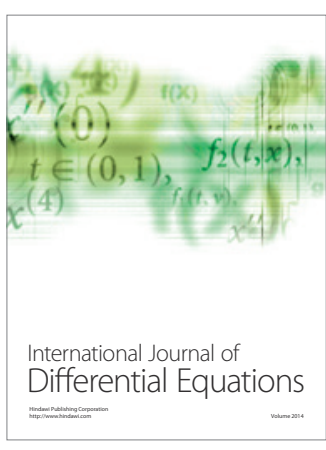
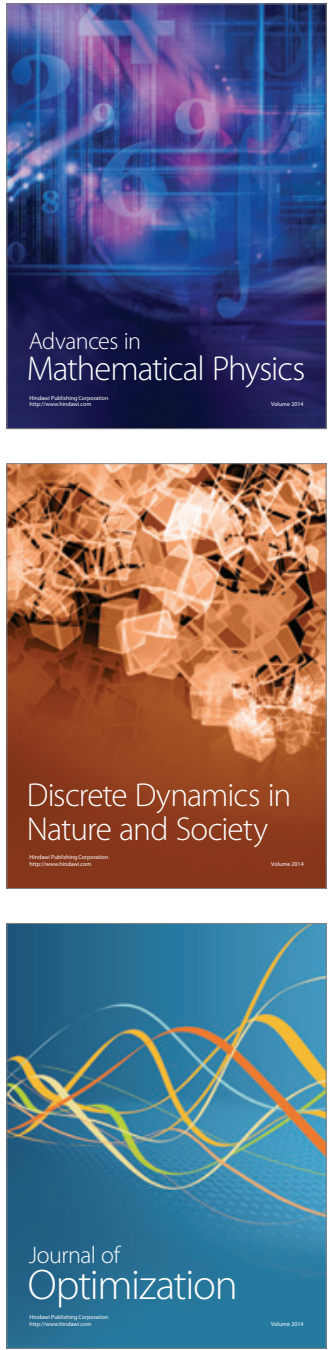